Int. J. Morphol.,

33(3):1176-1182, 2015

\title{
Impresiones 3D, Nueva Tecnología que Apoya la Docencia Anatómica
}

\author{
3D Impressions, New Technology that Supports Anatomical Teaching
}

\author{
Oscar Inzunza*; Iván Caro**; Germán Mondragón*; Felipe Baeza***; Álvaro Burdiles***** \& Guillermo Salgado*
}

INZUNZA, O.; CARO, I.; MONDRAGÓN, G.; BAEZA, F.; BURDILES, A. \& SALGADO, G. Impresiones 3D, nueva tecnología que apoya la docencia anatómica. Int. J. Morphol., 33(3):1176-1182, 2015.

RESUMEN: La dificultades de acceso al material cadavérico han forzado a los anatomistas a reemplazar la disección por el uso de preparaciones tratadas con diversos métodos de conservación y la utilización cada vez más frecuente de modelos anatómicos, elementos de alto costo que pretenden imitar, no siempre con éxito, a las preparaciones cadavéricas. En el último tiempo se han desarrollado métodos de impresión 3D de modelos de segmentos corporales utilizando imágenes de Tomografía Computadorizada o escáner de superficie, de modo que existe la tecnología para poder realizar réplicas de secciones corporales o de disecciones humanas para aplicarlas con fines docentes. En este trabajo presentamos nuestra experiencia en la tecnología de impresión 3D aplicada a la creación de réplicas de secciones corporales humanas, lo que nos ha permitido generar un símil de la sección corporal, real y tangible, que conserva exactamente las proporciones, las relaciones topográficas, la veracidad morfológica y el color, sin posibilidad de descomposición ni contaminación.

PALABRAS CLAVE: Impresión 3D; Réplicas anatómicas; Cadáveres; Secciones anatómicas; Educación médica.

\section{INTRODUCCIÓN}

"Hay tres modos de estudiar la anatomía, ya en los libros, ya en el cadáver, ó ya á beneficio de las figuras imitadas en láminas, cera, etc.” (Hurtado de Mendoza, 1829).

En los últimos tiempos la docencia para los cursos de anatomía se han visto seriamente afectada por el menguado acceso al material cadavérico, circunstancia paradojal en nuestro medio ya que si se aplicara la legislación nacional vigente, todos los Departamentos de Anatomía podrían contar con suficiente material anatómico (Libro $9^{\circ}$ del Código Sanitario -www.ispch.cl-). Esta situación ha forzado a los anatomistas a enfrentar de una manera distinta el desarrollo de sus programas. Así, en la mayoría de los cursos de anatomía se ha reemplazado la disección tradicional por la prospección de material cadavérico y el uso de preparaciones tratadas con diversos métodos de conservación (parafinado o plastinado). A esto se ha agregado, la utilización cada vez más frecuente de modelos anatómicos, elementos de alto costo que pretenden imitar - no siempre con éxito-a las preparaciones cadavéricas (Inzunza \& Salgado,
2011). El uso de estos recursos docentes se remontan al siglo XVI, en Zaragoza, como una forma de preservar en tercera dimensión las etapas de una disección anatómica compleja como, por ejemplo, la región axilar; ya que existía el convencimiento de que "las preparaciones frescas y naturales son sin disputa el mejor medio que se haya empleado hasta ahora para aprender anatomía” (Hurtado de Mendoza).

Ciertamente, no se puede pretender enseñar los temas anatómicos, al nivel de desempeño requerido para las carreras de medicina u odontología, sólo con preparaciones de segmentos corporales, modelos o fantomas. La utilización de estos recursos docentes muestra a los estudiantes una realidad sesgada, inexacta y ficticia. Está largamente probado que para el aprendizaje de temas morfológicos, el método consagrado por la historia es trabajo práctico con grupos pequeños, combinando el análisis de preparaciones cadavéricas con recursos imagenológicos (Chan et al., 2008). Además, se ha logrado demostrar que las actividades prácticas en anatomía tienen un efecto nivelador sobre las dife-

\footnotetext{
* Departamento de Anatomía, Facultad de Medicina, Pontificia Universidad Católica de Chile, Santiago, Chile.

** Facultad de Arquitectura y Diseño, Pontificia Universidad Católica de Chile, Santiago, Chile.

*** Facultad de Artes, Pontificia Universidad Católica de Chile, Santiago, Chile.

***** Departamento de Radiología, Facultad de Medicina, Pontificia Universidad Católica de Chile, Santiago, Chile. Financiado por Proyecto FONDEF CA13I10157
} 
rencias de origen (culturales, económicas, sociales) con que ingresan los estudiantes; ya que exponen de manera igualitaria a los educandos a claves visuales de calidad que son relevantes para el proceso de aprendizaje de los temas morfológicos (Norvis, 1971; Bravo \& Inzunza, 1995; Inzunza \& Bravo, 2002; Biasutto et al., 2006), facilitando la conceptualización tridimensional de los distintos órganos y sistemas (Granger, 2004). Este dato contingente no es menor, ya que apunta a las políticas de equidad que promueven las instituciones de educación superior. Lamentablemente, hoy en día, son cada vez más los encargados de cursos de anatomía — no necesariamente morfólogos - los que utilizan modelos o fantomas para la docencia anatómica.

En la década pasada Pommert et al. (2001) y más recientemente Shin et al. (2011) lograron crear, a partir de imágenes fotográficas de secciones transversales de un cuerpo humano, una reconstrucción virtual 3D que permite visualizar y reconocer los diferentes órganos y sistemas corporales. Este recurso computacional sin duda ofrece amplias aplicaciones en la docencia de la anatomía humana pero, mantiene el sesgo de la conceptualización volumétrica, resultando muy útiles para el profesional que ha tenido experiencia en la prosección o disección de un cuerpo humano, quien puede entonces hacer fácilmente la extrapolación al sujeto vivo. Sin embargo, para el novel alumno de las carreras del área médica la resolución del problema de la realidad volumétrica persiste, y no será resuelta hasta que el aprendiz se enfrente, aunque sea por una vez, al objeto real y tangible.

En el último tiempo se han desarrollado métodos de impresión estereolitográfica de modelos de segmentos corporales utilizando imágenes de Tomografía Computadorizada (Knox et al., 2005) o escáner de superficie (Hatamleh \& Watson, 2013); de modo que existe la tecnología para poder realizar réplicas 3D de secciones corporales o de disecciones humanas para aplicarlas con fines docentes. De hecho, recientemente esta tecnología se está incorporando al pabellón de anatomía (McMenamin et al., 2014). En este trabajo presentamos nuestra experiencia en la tecnología de impresión 3D aplicada a la creación de réplicas de secciones corporales humanas.

\section{MATERIAL Y MÉTODO}

Para la adquisición y procesamiento de imágenes se utilizaron dos secciones transversales de cadáveres humanos: una sección plastinada del tercio distal del brazo de, 2 cm de grosor; una sección fresca del tronco, de $3 \mathrm{~cm}$ de grosor, trazada a nivel de L1; preparaciones que forma parte de nuestro stock de recursos docentes; un segmento de co- lumna lumbar con la porción terminal de la aorta abdominal y un embrión de rata.

La sección plastinada de brazo y tronco fue escaneada en su superficie con el equipo Go!Scan 3D de la firma Creaform con una precisión de $0,1 \mathrm{~mm}$, recorriendo todas las superficies del corte.

Se fotografiaron en sus caras proximal y distal con una cámara Nikon D7000 Tiempo de exposición 5 s, apertura F32, ISO 100, en un ambiente de luz controlada (Pedrecshi \& Mery, 2006) con 8 tubos fluorescentes led de $860 \mathrm{~lm}$ con difuminadores.

Se adquirieron múltiples fotografías de forma instantánea de la sección con 52 cámaras digitales de 2 MG de resolución para hacer reconstrucción tridimensional (Jebara et al., 1999) con el sistema de fotogrametría con luz controlada. La reconstrucción se realizó utilizando el software Agisoft PhotoScan y el modelo se retocó utilizando el software Meshmixer.

La representación 3D de la sección Lumbar fue adquirida a partir de un Tomografia Axial Computarizada (TAC), en un equipo clínico GE Medical, con una representación de pixel de $1 \mathrm{~mm}$, considerada de forma heurística como óptimo, aunque el ideal para una reconstrucción es entre 0,3 y $0,6 \mathrm{~mm}$, reconstruyendo virtualmente los niveles L3 Y L4 junto con la porción terminal de la aorta abdominal, este archivo se procesó con el software Mimic's de Materialise, para ser fabricado en materiales de dos densidades, así representar tejidos rígidos y flexibles.

Para la representación del embrión se adquieren imágenes mediante el equipo Optical Projection Tomography (OPT) realizada en Bioptonics 3001 scanning microscope, marcado en superficie con DiI, con fluorescencia emitida en longitud de onda color rojo (Edgar, 2009). La imágenes fueron procesadas para reconstruir el modelo 3D mediante el software Mimic's de Materialise. El modelo 3D del embrión fue amplificado en $5 x$.

\section{Impresión 3D}

Se exploraron varias alternativas de impresoras existentes en el mercado, para seleccionar la más adecuada para cada caso (Tabla I, criterios de selección).

Se seleccionó la impresora Projet 660 Pro, con capacidad de fabricar detalles de $0,1 \mathrm{~mm}$, para la impresión de las prosecciones cadavéricas brazo plastinado y tronco fresco, esto debido a la amplia representación de colores que permite impresiones en base CMYK. 
Tabla I. Análisis comparativo de las distintas impresoras 3D utilizadas.

\begin{tabular}{lcccc}
\hline Máquina & Tipo de Impresión & Textura & Color & Materialidad \\
\hline Maquerbot & Extrución de Plástico & Insuficiente & 2 & Única \\
Eden 260v & Polyjet & Excelente & 1 & Única \\
Projet 3510 & Polyjet & Excelente & 1 & Única \\
Connex 500 & Multijet & Excelente & 45 & Múltiple \\
Projet $5500 \mathrm{X}$ & Colorjet & Excelente & 16 & Múltiple \\
Projet 460 & Colorjet & Buena & CMY & Única \\
Projet 660Pro & Colorjet & Buena & CMYK & Única \\
\hline
\end{tabular}

CMY= Cian, magenta, amarillo. CMYK= Cian, magenta, amarillo, negro.

Para la impresión de las vértebras lumbares L3, L4 y aorta, lo que se buscó fue representar la aorta en un material flexible y las piezas óseas e un material rígido, se seleccionó la maquina Projet 5500X, con capacidad de fabricar detalles de $32 \mu$, y que por sobre todo permite imprimir de forma simultánea con diferentes materiales rígidos y flexibles. También sería posible usar la máquina Connex 500.

Para la impresión del embrión la máquina que se seleccionó fue la Eden 260v dado que esta es la que presenta mejor resolución topográfica, con capacidad de fabricar detalles de $16 \mu$, y fácil acceso. Además, aquí podría ser utilizada la máquina la Projet 3510.

\section{RESULTADOS}

El seccionamiento del cadáver realizado a $-25^{\circ} \mathrm{C}$, utilizando una sierra de huincha con guía de corte, nos ha permitido cortar grandes bloques con un grosor regular. Para la sección de tronco fue de gran utilidad el scanner médico, ya que logramos maximizar el rendimiento docente de cada corte, mostrando los elementos anatómicos relevantes observables en cada cara de la sección del tronco.

La impresión 3D de la sección de brazo plastinado exhibe una alta similitud y proporcionalidad con el objeto real, llamando la atención que el post proceso de registro 3D logra una alta capacidad de reproducir la textura del corte plastinado. En términos de colores, se aprecia en la réplica una caída de los tonos, en especial de los colores amarillos, diferencia que ya se detecta en el archivo 3D (Fig. 1).

La impresión 3D de la sección de tronco fresco presenta una alta aproximación con el corte real; con el post proceso de registro 3D se logró optimizar la textura de la réplica, trabajo necesario considerando la textura más bien plana del corte fresco. Al analizar la fidelidad de colores también se aprecia aquí una caída de los tonos, en especial los matices amarillos y cafés (Fig. 2).

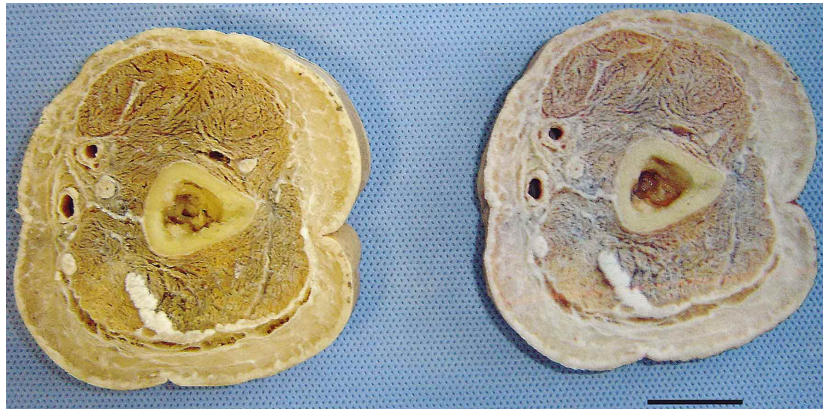

Fig. 1. Fotografías del corte transversal del tercio inferior del brazo plastinado con silicona (izquierda) y la réplica de la misma preparación realizada por impresión 3D, utilizando la impresora ProJet 660 Pro (derecha). En la réplica se aprecia que se mantienen la textura, la topografía y la proporcionalidad volumétrica; sin embargo, se advierte una caída en los tonos de los colores. Barra $=2,0 \mathrm{~cm}$.
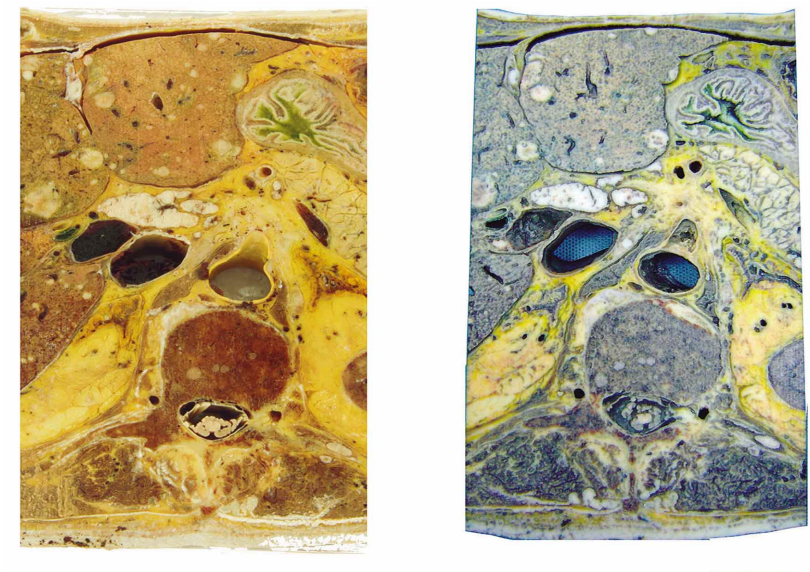

Fig. 2. Fotografía del corte de abdomen seccionado en fresco a nivel de L1-L2 (izquierda) y la réplica del corte realizado por impresión 3D. La réplica copia fielmente las características topográficas del original. Barra $=2,0 \mathrm{~cm}$.

La impresión 3D del segmento de columna lumbar y la porción terminal de la aorta abdominal muestra una alta correspondencia con la imagen tomográfica. Llama la aten- 
ción que el proceso de impresión 3D es capaz de reproducir el trabeculado óseo del cuerpo vertebral, tal como se demuestra en la radiografía tomada a la réplica. En la arteria aorta se visualizan dos placas de ateroma ubicadas en el origen de ambas arterias ilíacas comunes (Fig. 3).

En la impresión 3D magnificada a 5x del embrión de rata se observa que la réplica conserva los detalles y las proporciones del original; siendo esto especialmente notorio en los miembros y en las vibrisas (Fig. 4).

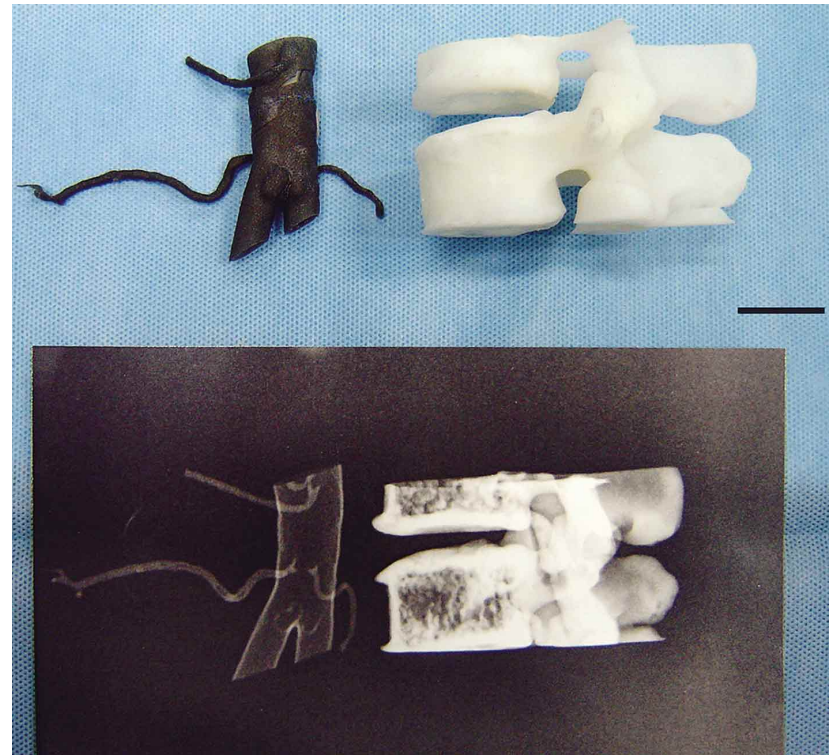

Fig. 3. Fotografía de la réplica del segmento lumbar de la columna vertebral y de la porción terminal de la aorta abdominal, obtenida a partir de una tomografía impresa en el equipo ProJet 5500X (arriba), y la imagen radiográfica de ambos elementos (abajo). En la radiografía se aprecia que la impresora es capaz de replicar el trabeculado de los cuerpos vertebrales y también puede copiar el lumen de los vasos sanguíneos. Barra $=2,0 \mathrm{~cm}$.

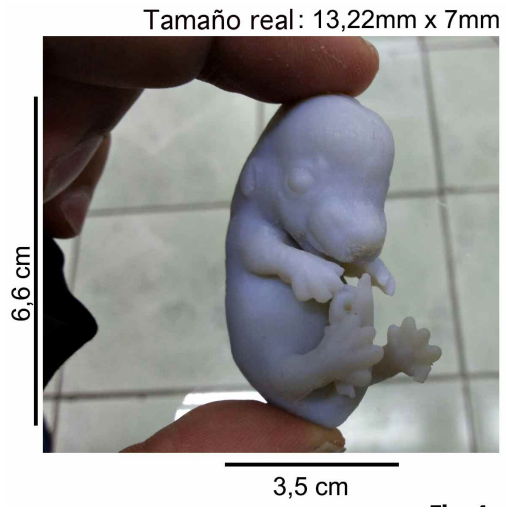

Fig. 4. Fotografía de una réplica de un embrión de rata reconstruido a partir de un registro OPT amplificada a 5X. En la impresión 3D se aprecia una alta definición de los detalles del original, especialmente la zona facial. Las dimensiones del original y de la replica magnificada aparecen en la imagen.

\section{DISCUSIÓN}

A nivel nacional e internacional, la combinación de disección y/o la prosección de material cadavérico sigue siendo el método fiable y confiable para el aprendizaje de anatomía. La actividad práctica, utilizando el cadáver humano como centro, constituye la única oportunidad que tiene el profesional sanitario en ciernes para confirmar empíricamente la situación de los órganos y sistemas corporales y su relación con la enfermedad y la muerte (Inzunza, 2008). Un sólido conocimiento anatómico, forjado en el trabajo práctico con material cadavérico, constituye la mejor preparación para que el aprendiz logre un buen desempeño en el examen clínico de sus pacientes y en la práctica de procedimientos en forma eficiente y segura; habilidades y destrezas que se han visto seriamente debilitadas en los alumnos de aquellas escuelas de medicina que abandonaron el trabajo anatómico con material cadavérico, tal como ocurrió en la Universidad de California en San Francisco, en la Universidad de California en Davis y en la Universidad de Washington, según el reporte de Rizzolo \& Stewart (2006).

Utilizando diferentes recursos tecnológicos para el registro y creación de archivos 3D: escaneo de superficie y tomografías, hemos demostrado que es posible crear réplicas de secciones transversales de distintos segmentos corporales (Figs. 1, 2 y 3). También hemos demostrado que la tecnología de impresión 3D permite crear réplicas magnificadas (Fig. 4) y reducidas en relación al objeto real, tal como lo demuestra en un trabajo reciente McMenamin et al. Estos autores han logrado producir réplicas de preparaciones anatómicas, de vasos sanguíneos y de senos peri nasales, a partir de un registro en Tomografía Computada (CT), método que encarece bastante el proceso (en Chile, una hora de CT está alrededor de U\$1.000). Nosotros estamos generando réplicas de secciones corporales humanas, altamente fidedignas, a partir de un escaneo de superficie (Figs. 1 y 2).

Nuestras réplicas mantienen una fidelidad de color con el original, la preparación plastinada o fresca, y el post proceso permite realzar la textura. En cambio el tratamiento que McMenamin et al., dan al tema del color de la impresión 3D es caricaturesco, manejando una paleta restringida a los colores utilizados generalmente en atlas y modelos anatómicos, asemejándose mucho más a estos que a la preparación real original que ellos utilizan. Ciertamente, en el cadáver las arteria no son rojas y los nervios no son amarillos; en la disección o prosección cadavérica las claves visuales relevantes para la identificación de estructuras son: la topografía, las relaciones, la textura, la consistencia, la forma y el color real de los elementos a identificar; sobre esto es que los anatomistas exigimos a nuestros alumnos para el reco- 
nocimiento de las diferentes estructuras anatómicas (Inzunza \& Salgado).

Asimismo, la fidelidad volumétrica de nuestras réplicas es notable, tema que es en extremo relevante para el aprendizaje de la anatomía seccional, ya que las tomografías, han introducido nuevas formas de ver la anatomía humana, presentando al cuerpo en secciones que pueden ser reconstruidas en 3D. Para el experto, la extrapolación desde la imagen computacional al sujeto vivo resulta fácil por la experiencia de los cursos de anatomía con preparados cadavéricos y por la vivencia clínica. Sin embargo, para los estudiantes de pregrado, el proceso de extrapolación de la pantalla o de la placa a la realidad clínica no resulta fácil; esto debido a que, desde un tiempo a esta parte, los cursos de anatomía han comenzado a reemplazar el análisis de preparaciones cadavéricas por el estudio del cuerpo humano en los modelos y software. Actualmente, en nuestro país están disponibles modelos plásticos de alto costo, desarrollados como respuesta a la evidente escasez de muestras de secciones cadavéricas. Sin embargo, estos modelos anatómicos no logran una adecuada fidelidad con la realidad en términos de formas, colores, tamaños y detalles anatómicos; características que resultan fundamentales para el aprendizaje de la anatomía seccional (Inzunza et al., 2003, 2007).

Las máquinas usadas para la réplica de los cortes fueron ProJet 460 y ProJet 660 Pro, las dos con capacidad de imprimir un detalle de $0,1 \mathrm{~mm}$, la diferencia fundamental está en la aplicación de color, la primera genera una paleta de colores con 3 tintas CMY (cian, magenta y amari1lo), la segunda con CMYK; que incluye el color negro lo que permite alcanzar una mayor cantidad y calidad de tona- lidades e intensidad de color. Los resultados en la ProJet 460 se distancian de las tonalidades reales, los resultados de la ProJet 660 Pro son considerados óptimos para reconocer las distintas estructuras de los cortes anatómicos. Las impresoras de la línea 600 generan réplicas rígidas, en un material que es un tipo de yeso plastificado, de alta resistencia y con una fineza de detalles que reproduce perfectamente la textura de los cortes anatómicos (Figs. 1 y 2).

El equipo utilizado para imprimir el archivo de la columna lumbar fue la ProJet 5500X, de la firma 3D System, que imprime en material plástico de distinta consistencia; así, las vértebras se imprimieron en resina rígida y la arteria aorta en una resina de consistencia flexible. Esta impresora permite controlar la variable de rigidez del producto. A este respecto debemos señalar que nuestro prototipado de aorta resultó relativamente feble y se desgarró; sin embargo, este material permitió ser suturado, sirviendo como un buen modelo para sutura vascular (Dr. Javier Román V., comunicación personal). Llama la atención que la fidelidad de detalles del equipo permite mantener el lumen de las ramas pequeñas de la aorta, tal como se aprecia en la radiografía (Fig. 3). Lamentablemente, este equipo ofrece pocas alternativas de colores (blanco, negro, gris y azul), no combinables, a diferencia de las impresoras de la línea 660.

La réplica ampliada del feto de ratón se imprimió en un equipo Eden 260v, de la firma Stratasys; dispositivo que imprime en resina curada con luz ultravioleta, ofreciendo una textura lisa y brillante, que copia a la perfección los detalles de la superficie del modelo original. Lamentablemente, este equipo ofrece pocas alternativas de color, todas en tonos sólidos (Fig. 4).

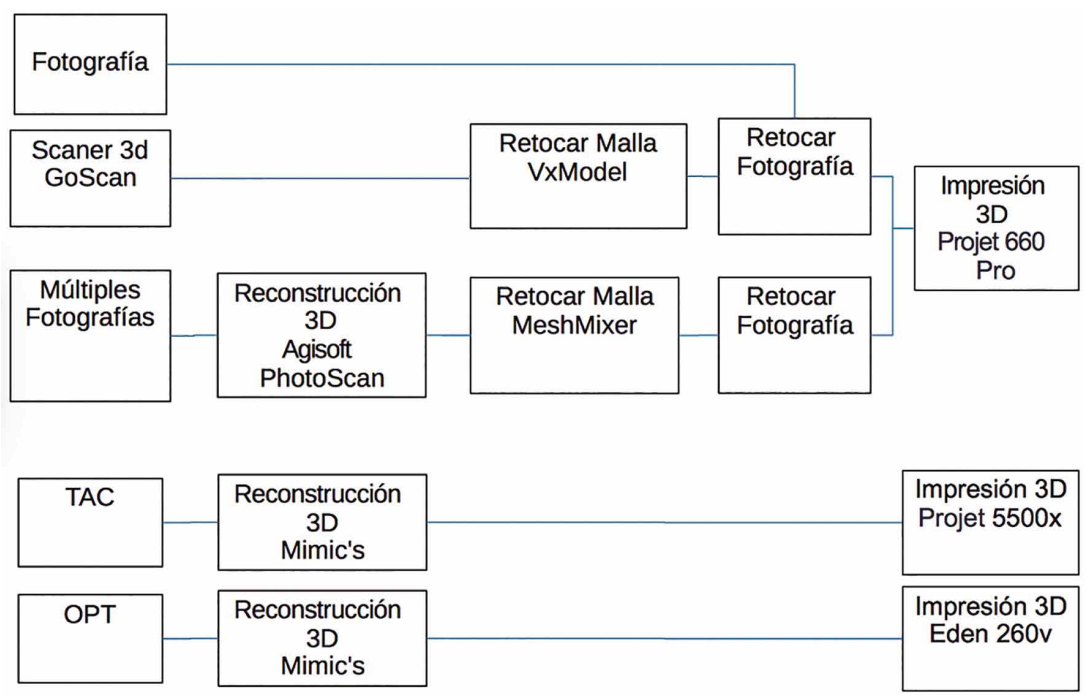

Fig. 5. Esquema que resume el proceso de registro, post procesamiento e impresión utilizado para distintas piezas replicadas. 
En este trabajo demostramos que a partir de estas secciones corporales es posible crear un registro en imágenes $2 \mathrm{D}$ y $3 \mathrm{D}$, combinando procedimientos fotográficos y de escáner de superficie y desarrollar un sistema procedimental que defina y agote las variables de proporcionalidad, color, textura, relieve y forma, permitiendo crear impresiones 3D tomando como base el registro de las secciones corporales (Fig. 5). Con la réplica física se cuenta con un símil de la sección corporal, real y tangible, que conserva exactamente las proporciones, las relaciones topográficas, la veracidad morfológica y el color, sin posibilidad de descomposición ni contaminación. Por otro lado, esta solución permitiría la accesibilidad a la realidad humana evitando los conflictos de disponibilidad, bioseguridad, culturales y religiosos que pudiesen presentarse, sin ir en desmedro de la formación del estudiante y/ o profesional, al contar con una réplica exacta, evitando el sesgo hacia una realidad burda y ficticia de los modelos anatómicos. Por sus características, estas herramientas docentes permitirán la transferencia del conocimiento anatómico desde las réplicas anatómicas al reconocimiento práctico de estructuras en imágenes médicas, tomografías computarizadas y resonancia nuclear magnética; beneficiándose con este material didáctico los alumnos de las carreras del área médica, quienes serán los futuros usuarios de estas técnicas imagenológicas de amplia difusión.

Entonces, pasadas casi dos centurias, podemos agregar a la sentencia de Hurtado de Mendoza otro recurso docente a los que él menciona: libros, cadáver, figuras imitadas $\mathrm{y}$, ahora, en el siglo XXI, impresiones 3D de preparaciones anatómicas.

\section{AGRADECIMIENTOS}

A la Dra. Marcia Gaete y Dr. Alasdair Edgar, Craniofacial Development and Stem cell Biology Department, King's College London. María Jesus Musura, Protaico. Eduardo Guiloff, Espacio 3D. Sebastián Oportus, BetaWorks.

INZUNZA, O.; CARO, I.; MONDRAGÓN, G.; BAEZA, F.; BURDILES, A. \& SALGADO, G. 3D impressions, new technology that supports anatomical teaching. Int. J. Morphol., 33(3):1176-1182, 2015.

SUMMARY: The difficulties of access to cadavers have forced anatomists to replace dissections with the use of preparations treated with various methods of conservation and/or anatomical models; high-cost items that intended to imitate - not always successfully - the cadaveric preparations. Recently, new methods have been developed for 3D printing of models of body segments using computed tomography images or scanner surface, so that the technology exists to create replicas of body sections or cadaver dissections to apply for teaching purposes. We present our experience in 3D printing technology applied to create replicas of human body sections, allowing us to generate an accurate reproduction, with real and tangible sections which retain the proportions exactly as well as topographic relations, morphological and color accuracy, without the possibility of decomposition or contamination.

KEY WORDS: 3D printing; Morphology; Anatomical teaching; Anatomical models.

\section{REFERENCIAS BIBLIOGRÁFICAS}

Biasutto, S. N.; Caussa, L. I. \& Criado del Río, L. E. Teaching anatomy: cadavers vs. computers? Ann. Anat., 188(2):187-90, 2006.

Bravo, H. \& Inzunza, O. Evaluación de algunos programas computacionales en la enseñanza de la anatomía y neuroanatomía de la Facultad de Medicina de la Pontificia Universidad Católica de Chile. Rev. Chil. Anat., 13(1):79-86, 1995.

Chan, L. K. \& Ganguly, P. K. Evaluation of small-group teaching in human gross anatomy in a Caribbean medical school. Anat. Sci. Educ., 1(1):19-22, 2008.

Edgar, A. An Optical Projection Tomography Scanning Microscope. infocus Magazine, 15:5-16, 2009.
Granger, N. A. Dissection laboratory is vital to medical gross anatomy education. Anat. Rec. B New Anat., 281(1):6-8, 2004.

Hatamleh, M. M. \& Watson, J. Construction of an Implant-Retained Auricular Prosthesis with the Aid of Contemporary Digital Technologies: A Clinical Report. J. Prosthodont., 22(2):132-6, 2013.

Hurtado de Mendoza, M. Tratado elemental completo de anatomía general ó [sic] fisiológica, de anatomía especial ó [sic] descriptiva, de anatomía de regiones ó [sic] quirúrgica y de anatomía patológica ó [sic] médica con arreglo al estado actual de esta ciencia y progresos que ha hecho en estos últimos años. Tomo 1. Madrid, Imprenta que fue de Garcia, 1829.

Inzunza, O. Competencias Generales en Medicina, Rol de la Anatomía. Int. J. Morphol., 26(2):243-6, 2008. 
Inzunza, O. \& Bravo, H. Animación computacional de fotografías, un real aporte al aprendizaje práctico de anatomía humana. Rev. Chil. Anat., 20(2):151-7, 2002.

Inzunza, O.; D'Acuña, E. \& Bravo, H. Evaluación práctica de anatomía. Rendimiento de los alumnos de primer año de medicina ante distintas formas de preguntar. Int. J. Morphol., 21(2):131-6, 2003.

Inzunza, O. \& Salgado, G. Evaluaciones Prácticas Objetivadas en Anatomía: Diferencias de Rendimiento en Preguntas Realizadas en Modelos, Preparaciones Anatómicas y Cadáveres. Int. J. Morphol., 29(2):490-5, 2011.

Inzunza, O.; Vargas, A. \& Bravo, H. Anatomía y neuroanatomía, disciplinas perjudicadas por la reforma curricular. Int. J. Morphol., 25(4):825-30, 2007.

Jebara, T.; Azarbayejani, A \& Pentland, A. 3d structure from 2d motion. IEEE Signal Proc. Mag., 16(3):66-84, 1999.

Knox, K.; Kerber, C. W.; Singel, S. A.; Bailey, M. J. \& Imbesi, S. G. Rapid Prototyping to Create Vascular Replicas From CT Scan Data: Making Tools to Teach, Rehearse, and Choose treatment strategies. Catheter. Cardiovasc. Interv. 65(1):4753, 2005.

McMenamin, P. G.; Quayle, M. R.; McHenry, C. R. \& Adams, J. $\mathrm{W}$. The production of anatomical teaching resources using three-dimensional (3D) printing technology. Anat. Sci. Educ., 7(6):479-86, 2014.

Norvis, G. Didáctica y estructura de los medios audiovisuales. Buenos Aires, Kapeluz, 1971.

Pedreschi, F.; León, J.; Mery, D.; Moyano, P. Development of a computer vision system to measure the color of potato chips. Food Res. Int., 39(10):1092-8, 2006.

Pommert, A.; Höhne, K. H.; Pflesser, B.; Richter, E.; Riemer, M.; Schiemann, T.; Schubert, R.; Schumacher, U. \& Tiede, U. Creating a high-resolution spatial/symbolic model of the inner organs based on the Visible Human. Med. Image Anal., 5(3):221-8, 2001.

Rizzolo, L. J. \& Stewart, W. B. Should we continue teaching anatomy by dissection when ...? Anat. Rec. B New Anat., 289(6):215-8, 2006.

Shin, D. S.; Park, J. S.; Shin, B. S. \& Chung, M. S. Surface models of the male urogenital organs built from the Visible Korean using popular software. Anat. Cell Biol., 44(2):151-9, 2011.

\author{
Dirección para Correspondencia: \\ Prof. Dr. Oscar Inzunza \\ Departamento de Anatomía Normal \\ Escuela de Medicina \\ Pontificia Universidad Católica de Chile \\ Santiago \\ CHILE
}

Email: oinzunza@med.puc.cl

Recibido : 11-03-2015

Aceptado: 27-06-2015 